\title{
Journal hijacking an imminent threat Journal hijacking an imminent threat
}

\section{Introduction}

Hijacking in the real terms means 'To take control of (something) without permission or authorization and use it for one's own purposes". Hijacking in the real world has not remained limited to materials but now have encroached the research world as well, seriously compromising and challenging the reliability of the scientific data. This phenomenon came to light in early 2012 when the online hijackers started creating counterfeit websites which mimic the legitimate websites of renowned journals. ${ }^{1}$ The fake websites are carefully engineered and fine tuned to mimic the genuine points, like title of the genuine/authentic original journal, the impact factors, postal address, list of the journal's editorial board and even the international standard serial number (ISSN). ${ }^{2}$ They provide a link from a fake website to the authentic journal's profile in the master journal list of Thomson Reuters. Sometimes, authors are aware that there should be a link between the Thomson Reuters' and the journal's website, but they would not notice that this link should be from Thomson Reuters to the Journal, not from the Journal to Thomson Reuters. ${ }^{3}$

Emails are sent under the "call for papers" to various researchers with the incentive of rapid processing and acceptance of articles within period of time ranging from few days to 2 weeks. Usually journals with not very high impact factors, those with individual publisher with single journal, being published in non English language are being targeted. Well known journals like "Vulfenia Journal", "Archives des Sciences,", Emergencias (an emergency medicine journal from Spain), the Journal of the American Medical Association (JAMA), Vitae-Revista (The Official Publication of the Faculty of Pharmaceutical Chemistry at the University of Antioquia, Colombia), Amala (published by the Amala Cancer Research Center in India), TERAPEVTICHESKII ARKHIV (from Russia), Kardiologiya (from Russia) and Revue Scientifique et Technique (published by the World Organization for Animal Health) have been the recent victims of the same. $^{4}$

During the last few years, there has been dramatic increase in such incidences raising the number to nearly 64 journals as on June 2015 . (http://scholarlyoa.com/other-pages/hijacked-journals) Such articles are published without the necessary peer review therefore/ thus compromising the reliability and validity of the scientific information subsequently affecting the outcomes of systematic reviews misleading the decision makers like/may it be the clinicians, academicians, researchers or policy makers. ${ }^{4}$ There is an urgent need to sensitise and train the stakeholders involved in identifying such websites
Volume I Issue I - 2015

\author{
Snehal Patil,' Tatyasaheb Patil, ${ }^{2}$ Siddhi \\ Hathiwala ${ }^{3}$ \\ 'Department of Public Health Dentistry, Krishna Institute of \\ Medical Sciences Deemed University, India \\ ${ }^{2}$ Department of Pharmacology, Bharati Vidyapeeth Medical \\ College and Hospital, India \\ ${ }^{3}$ Department of Public Health Dentistry, Guru Govind Singh \\ College of Dental Science and Research Centre, India
}

Correspondence: Snehal Patil,Assistant professor, Department of Public Health Dentistry School of Dental Sciences Krishna Institute of Medical Sciences Deemed University, Karad, Maharashtra, India, Tel 9764I36089, Email snehal_2086@yahoo.com

Received: June 25, 2015 | Published: July 04, 2015

so as to avoid being victimized by the cyber criminals. In years to come various organizations like International Committee of Medical Journal Editors, World Association of Medical Editors and Committee on Publication Ethics would be able to contribute significantly in the anti hijacking strategy.

\section{Acknowledgements}

None.

\section{Conflict of interest}

The author declares no conflict of interest.

\section{References}

1. Butler D. Sham journals scam authors. Nature. 2013;495(7442):421122.

2. Kolahi J, Khazaei S. Journal hijacking: A new challenge for medical scientific community. Dental Hypotheses. 2015;6(1):3-5.

3. Jalalian M, Mahboobi H. Hijacked journals and predatory publishers: Is there a need to re-think how to assess the quality of academic research? Walailak Journal of Science and Technology. 2014;11(5):389-394.

4. Jalalian M. Hijacked journals are attacking the reliability and validity of medical research. Electron Physician. 2014;6(4):925-926. 